\title{
Negative thermal diffusivity enhancement in semiconductor nanofluids
}

\author{
Martha Patricia González-Araoz ${ }^{1,2}$, José Francisco Sánchez-Ramírez ${ }^{3^{*}}$, \\ José Luis Jiménez-Pérez ${ }^{3}$, Ernesto Chigo-Anota ${ }^{4}$, José Luis Herrera-Pérez ${ }^{3}$, \\ Julio Gregorio Mendoza-Álvarez ${ }^{5}$ \\ ${ }^{1}$ Center of Applied Biotechnology Research, National Polytechnic Institute (CIBA-IPN), Tlaxcala, Mexico \\ ${ }^{2}$ Faculty of Engineering, Autonomous University of Puebla (BUAP), Puebla, Mexico \\ ${ }^{3}$ National Polytechnic Institute UPIITA-IPN, Distrito Federal, México; ${ }^{*}$ Corresponding Author: jfsanchez@ipn.mx \\ ${ }^{4}$ Chemical Engineering Faculty, Autonomous University of Puebla (BUAP), Puebla, Mexico \\ ${ }^{5}$ Physics Department, CINVESTAV-IPN, Distrito Federal, Mexico
}

Received 3 September 2012; revised 5 October 2012; accepted 18 October 2012

\begin{abstract}
Colloidal suspensions of semiconductor InP@ZnS nanoparticles were prepared using single-step procedure without precursor injection. Thermal properties of toluene containing InP@ZnS semiconductor with different sizes $(3.1,4.2$, and 4.6 $\mathrm{nm}$ ) were measured by mode mismatched dualbeam thermal lens technique. This was done in order to measure the effect of the presence of semiconductor nanoparticles and size on the nanofluids thermal diffusivity. The characteristic time constant of the transient thermal lens was estimated by fitting the experimental data to the theoretical expression for transient thermal lens. The thermal diffusivity of the nanofluids (toluene, containing InP@ZnS semiconductor nanoparticles) it seems to be strongly dependent on the presence of semiconductor nanoparticles and particles size. For the case of nanofluids consisting of InP@ZnS nanoparticles dispersed in toluene, it was observed a decrease in the thermal diffusivity. Such behavior differs from other nanofluids, in the sense that they had shown positive thermal diffusivity enhancement. The minimum diffusivity was achieved for the nanoparticles with lowest size. Plausible explanation for such nanofluids low thermal diffusivity with semiconductor nanoparticles is given. UV-Vis spectroscopy, TEM and high-resolution electron microscopy (HRTEM), and energy dispersive spectroscopy (EDS) techniques were used to characterize the InP@ZnS nanoparticles.
\end{abstract}

Keywords: Thermal Diffusivity; Nanofluids;

Semiconductor Nanoparticles; Thermal Lens

\section{INTRODUCTION}

After their thermal properties variations were reported at the end of last century [1], nanofluids are new compound liquids that had obtained much interest. Small amount $(<1 \%$ volumetric fraction) of nanoparticles has significantly increased thermal conductivity of liquids such as water, ethylene glycol, and oil when they are dispersed in these liquids. Although research studies on the thermal transport properties of nanofluids have been extensively performed [2-5], these have been limited to study of the enhanced thermal conductivity of nanofluids containing different types of nanoparticles such as oxides, pure metallic and carbon nanotubes using different techniques [6-11]. Thermal diffusivity is key for evaluating their thermal performance under flow conditions and in convective heat transfer-based applications, and despite this very few efforts have been made to determine it. In recent years, we have seen an increasing interest in the measurement and modeling of the thermal diffusivity properties in fluid suspensions of nanometer-sized metallic particles [12-15]. However, semiconductor nanoparticles [16] are expected to significantly modify nanofluids thermal transport behavior due the ability to tailoring their structure and nanometer size. The interesting physic and chemical properties of semiconductor nanoparticles result from their structure and nanometer size $[17,18]$.

Nanofluids containing semiconductor nanoparticles are important candidates for applications such as DNA tagging or aqueous monitoring for biological and chemical changes in a given environment, targeted thermal agents in medical therapies with extended precision of thermal effects below cellular dimensions [19,20]. Provide the opportunity to study the influence of semiconductor nanoparticles (with sizes of the same order as the phonon mean free path) on the thermal diffusivity $(D)$ of 
liquids. A dramatic decrease in the thermal conductivity of semiconductor nanoparticles as the particle size becomes of the same order as the phonon mean free path, has been very recently observed [21,22]. Also, to our knowledge, there is no study on the thermal diffusivity of nanofluids containing InP@ZnS semiconductor nanoparticles.

In this article, effect of the presence of semiconductor nanoparticle and size of InP covered with $\mathrm{ZnS}$ shell on the thermal diffusivity of nanofluids was studied. Thermal diffusivity measurements are carried out in toluene containing core-InP/shell-ZnS nanoparticles using the Thermal Lens Spectroscopy (TLS) [23]. This technique provides reliable photothermal alternative for measuring thermal diffusivities of semiconductor materials with high sensitivity. We used the mode mismatched dualbeam thermal lens technique to measure the thermal diffusivity of nanofluids.

\section{EXPERIMENTAL DETAILS}

\subsection{Sample Preparation}

Using single-step procedure without precursor injection [24] colloidal dispersion of InP@ZnS nanocrystals was synthesized and then stored in glovebox. Firstly the precursor of indium (indium myristate, In(MA)) was prepared, $0.1 \mathrm{mmol}$ of indium acetate $\left(\operatorname{InAc}_{3}\right)$ were mixed under inert atmosphere with $0.3 \mathrm{mmol}$ of myristic acid and $5.0 \mathrm{~g}$ of 1-octadecene (ODE) in a $50 \mathrm{~mL}$ threeneck flask equipped with condenser. The mixture was heated at $100^{\circ} \mathrm{C}$ during $1 \mathrm{~h}$ under vacuum to obtain an optically clear solution, backfilled with Ar gas, and cooled down at room temperature.

In another $50 \mathrm{~mL}$ three-neck flask equipped with condenser, $0.2 \mathrm{mmol}$ of zinc stearate, $0.2 \mathrm{mmol}$ of dodecanethiol (DDT), $0.2 \mathrm{mmol}$ of tris (trimethylsilyl) phosphine $\left(\mathrm{P}(\mathrm{TMS})_{3}\right), 10 \mathrm{~mL}$ of ODE and different contents of In (MA) $(0.1,0.2,0.3,0.4 \mathrm{mmol}$, sample S1, S2, S3 and $\mathrm{S} 4$, respectively) were stirred under inert atmosphere. The mixture was then heated at $200^{\circ} \mathrm{C}$ of temperature for $1 \mathrm{~h}$ and the temperature was then decreased at room temperature. The colloidal dispersions thus prepared are stable, with $3.1-4.6 \mathrm{~nm}$ in average diameter. The nanocrystals were repeatedly purified with chloroform/ methanol/ acetone (1:1:10 vol:vol:vol) followed by centrifugation, to remove the starting materials and side products.

In order to obtain nanofluids containing InP@ZnS nanoparticles, the colloidal dispersion were subsequently dried in vacuum and the semiconductor nanoparticles were dissolved pre-weighed quantities in toluene maintaining the same concentrations $(2.5 \% \mathrm{w} / \mathrm{w})$ and were placed in a quartz cuvette of $1 \mathrm{~cm}$ thick for the optical and thermal measurement. All the experiments were performed at room temperature and were subjected to ultrasonic processing prior to each measurement.

\subsection{Characterization}

Room-temperature optical absorption spectra of the colloidal samples were recorded using a $10 \mathrm{~mm}$ path length quartz cuvette in an UV-Vis-NIR scanning spectrophotometer (Shimadzu UV 3101PC double beam). For electron microscopy analysis, two microscopes, Jeol JEM200 and Tecnai 200 TEM, were used for the lowmagnification and high-resolution observations of the samples, respectively. High-resolution electron microscope (HRTEM) images were digitally processed by using filters in the Fourier space. For transmission electron microscopy (TEM) observations, a drop of colloidal solution was spread on formvar carbon deposited on copper microgrid and dried in vacuum. For composition analysis, energy dispersive spectroscopy (EDS) of the samples performed using a Jeol JSM6390 scanning electron with NORAN analytical system attached.

\subsection{Theory of Thermal Lens}

The TL effect of such nanofluids was based on their laser-induced heating and time resolved monitoring on the thermal effects. The schematic diagram of the TL experimental setup is shown in Ref. [25]. The TL effect is caused by the deposition of heat via non-radiative decay processes after the laser beam, with Gaussian profile, has been absorbed by the sample. In this situation a transverse temperature profile, $\Delta T(r, t)$, is established. The temporal evolution $\Delta T(r, t)$ is scaled according to characteristic time constant:

$$
t_{c}=\omega_{e}^{2} / 4 D
$$

where $\omega_{e}$ is the excitation laser beam radius at the sample and $D$ is the thermal diffusivity. Owing to $\Delta T(r, t)$, a temperature coefficient of the optical path length change, $\mathrm{d} s / \mathrm{d} T=\mathrm{d} n / \mathrm{d} T$ (for liquids), is generated, creating a lens-like optical element: the so-called TL effect. The propagation of a probe laser beam through this TL results in a variation of its on-axis intensity, $I(t)$, which can be calculated using diffraction integral theory. In transient regime, an analytical expression can be obtained for the probe beam intensity, $I(t)[26]$ :

$$
=I(0)\left[1-\frac{\theta}{2} \tan ^{-1}\left(\frac{2 m V}{\left[(1+2 m)^{2}+V^{2}\right] \frac{t_{c}}{2 t}+1+2 m+V^{2}}\right)\right]^{2}
$$

where:

$$
m=\left(\frac{\omega_{p}}{\omega_{e}}\right)^{2} ; \quad V=\frac{Z_{1}}{Z_{c}} ; \quad \theta=-\frac{P_{e} A_{e} l_{0}}{k \lambda_{p}}\left(\frac{\mathrm{d} n}{\mathrm{~d} T}\right)_{p} ;
$$


in Eq.2, $I(0)$ is the initial value of $I(t)$; $\theta$ is the thermally induced phase shift of the probe beam after passing through the sample; $Z_{c}(12.89 \mathrm{~cm})$ is the confocal distance of the probe beam and $Z_{1}(8.0 \mathrm{~cm})$ is the distance from the probe beam waist to the sample. $\omega_{e}\left(\omega_{p}\right)$ is the spot size of the excitation (probe) laser beam at the sample; $k(D)$ is the thermal conductivity (diffusivity) of the sample; $P_{e}$ is the incident power; $A_{e}$ is the optical absorption coefficient at the excitation beam wavelength $\lambda_{e}$; and $\mathrm{d} n / \mathrm{d} T$ is the temperature dependence of the sample refractive index. The so-called characteristic time of TL $t_{c}$, is defined as $D=\omega_{e}^{2} / 4 t_{c}$ with $D=k / \rho c$ where $\rho$ is the density and $c$ is the heat capacity of the nanofluid. The experimental value of the beam waist of the excitation laser beam is $\omega_{e}=40 \mu \mathrm{m}$. Eq. 2 describes the time resolved transient that creates the TL effect. Then by fitting the equation of $I(t)$ (Eq.2) to the experimental data, as a function of time $(t)$, it is possible to obtain the sample thermal diffusivity $D$ from $t_{c}$ as adjustable parameter.

\section{RESULTS AND DISCUSSION}

To characterize the particles, TEM, and HRTEM, EDS were used along with optical absorption spectroscopy to study the size, size distribution, composition, and fine structure of semiconductor InP@ZnS colloids formed at different contents of In (MA).

The effects of In (MA) content in the semiconductor InP@ZnS colloidal nanoparticles can be clearly observed in Figure 1, which shows transmission electron micrographs with their corresponding size distribution histograms for each of the samples. From this figure, we can observe that the particle shape was nearly spherical with sizes in the nanometer scale. The size distributions followed a Gaussian fit with narrow size distributions. Appearance of no particles in the case of S1 sample (not submitted) indicates the formation of neither type of nanoparticles. Absence of bimodal distribution in the size distribution histograms suggests that the nanoparticles obtained at different contents of In (MA) correspond to a formation of nanoparticles containing InP-ZnS and not to physical mixture of nanoparticles of $\mathrm{InP}$ and $\mathrm{ZnS}$. The average particle size increased with the increase of the molar concentration of In (MA) in semiconductor nanoparticles. So, the content of In (MA) could control the formation and size of the semiconductor nanoparticles.

For closer observation on the crystallinity, and fine structure of the semiconductor nanoparticles, HRTEM images of three samples were recorded. From the HRTEM images shown in Figure 2, we can observe the formation of well-defined crystalline nanoparticles for three samples. Interplanar spacing calculated for both samples corresponds well with the interplanar spacing of the corresponding indium phosphide structures of the zinc-
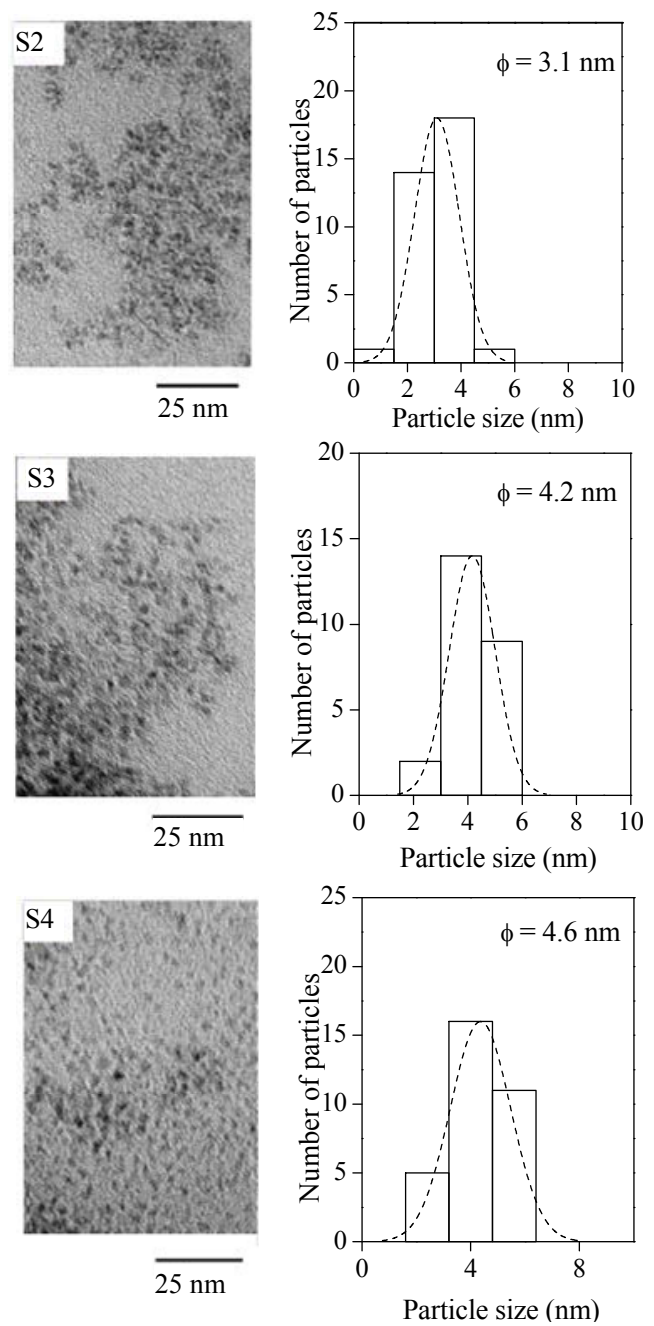

Figure 1. Electron micrographs and normalized size distribution of particles of the semiconductor nanoparticles with different contents of In (MA). Average particles size $(\phi)$ is calculated from Gaussian fitting of the histograms: $\phi_{S 2}=3.1 \mathrm{~nm}, \phi_{S 3}=$ $4.2 \mathrm{~nm}$ and $\phi_{S 4}=4.6 \mathrm{~nm}$.

blende phase. We tried to analyze each discrete particle in order to observe the possible presence of $\mathrm{ZnS}$ shell; however, it was not possible to confirm the presence of $\mathrm{ZnS}$ on the surfaces of InP from their HRTEM images, which possibly means that the shell is too thin to detect.

To confirm the presence of the ZnS, EDS analysis on the particles was carried out inside the transmission electron microscope for three sets of samples: S2, S3 and S4. We could not detect any particles of monocomponent nature of InP or $\mathrm{ZnS}$ in them. Though the composition of individual particles varied from particle to particle for a given sample, the presence of In, P, Zn and S always was confirmed.

The global composition of the samples was also monitored through EDS spectroscopy in a scanning electron microscope. In Figure 3, EDS spectra of the sample S3 


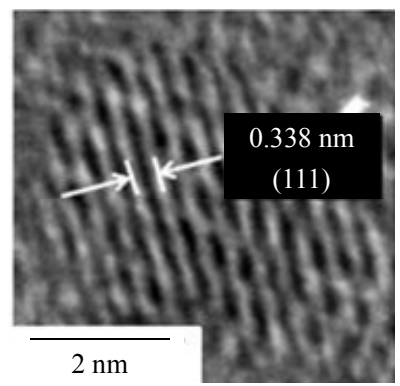

(a)

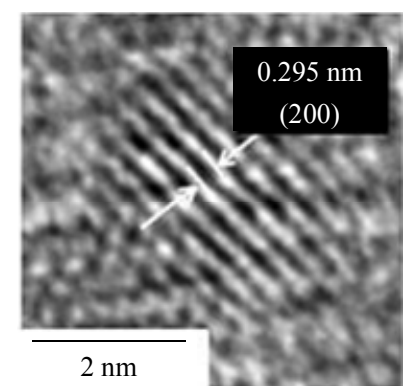

(b)

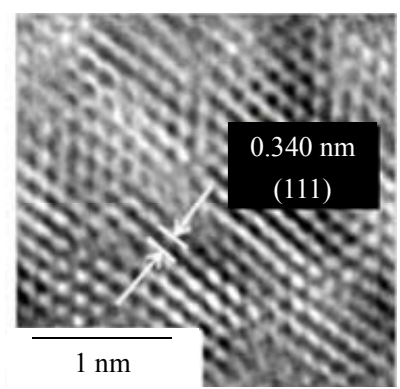

(c)

Figure 2. Typical HRTEM images of semiconductor nanoparticles with phase zinc-blende: (a) for S2 with lattice spacing of $0.338 \mathrm{~nm}$; (b) for S3 with lattice spacing of $0.295 \mathrm{~nm}$; and (c) for S4 with lattice spacing of $0.340 \mathrm{~nm}$.

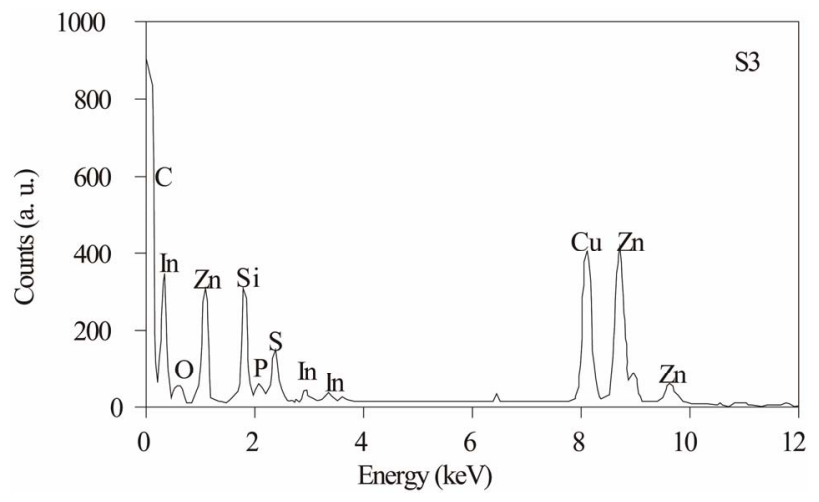

Figure 3. EDS spectra of the colloidal samples for S3.

is presented. The presence of $\mathrm{Zn}$ and $\mathrm{S}$ was clearly confirmed. Along with the In, P, Zn and S emission peaks, there appeared carbon, oxygen and copper emissions in the EDS spectra of the samples. While the origin of the carbon, oxygen, and copper emissions is related to the formvar carbon film on the grid surface and copper microgrid, the atomic ratio of In:P and $\mathrm{Zn}: \mathrm{S}$ was calculated to be more or less 1:1. Similar results were obtained for samples $\mathrm{S} 2$ and $\mathrm{S} 4$.

Formation of semiconductor nanoparticles was also confirmed from the UV-Vis absorption spectra of the samples. Nanoparticles such as InP in the nanoscale strongly absorb light when the excitation energy is greater than the bandgap energy. During this process, electrons are promoted from the valence to the conduction band.

Measurements of UV-Vis spectra reveal a large number of energy states in the semiconductor nanoparticles [27]. The lowest excited energy state is shown by the first observable peak in the absorption spectrum, known as the quantum-confinement peak. The energy of the quantum-confinement peak depends on the size, shape and structure core@shell [28]. Figure 4 shows the absorption spectra of colloidal solutions containing InP@ZnS particles obtained at different contents of In (MA). We show the absorption spectra for samples S2,
S3 and S4, all spectra were recorded at room temperature. From the figure, we can observe that an absorption shoulder appears at 496, 508 and $516 \mathrm{~nm}$ for samples S2, S3 and S4 respectively. These absorption results show that as In (MA) content increases, there is a shift to lower energies of the bandgap energy corresponding to transitions between the heavy-hole ground state in the valence band to the electron ground state in the conduction band due to the quantum confinement effect. This bandgap red shift confirm that the particle semiconductor size increased with the increase of In (MA) contents in the synthesis. These results are consistent with those obtained by TEM.

The time evolution of TL signal for the fluids containing semiconductor nanoparticles is shown in Figure 5, where the symbols represent the experimental data points (o) and the solid lines correspond to their best fits to Eq.1 leaving $\theta$ and $t_{c}$ as adjustable parameters. Using $t_{c}=\omega_{e}^{2} / 4 D$ and having $\omega_{e}$, we obtained $D$ for the corresponding nanofluids. It is possible to see from figure that the TL signal decreases as time elapses, indicating that the thermal lens are divergent, thus defocusing the probe beam on the detector. This behavior is because the temperature coefficient of the optical path length $\mathrm{d} s / \mathrm{d} T$, is negative for most of the transparent liquids and semitransparent materials as the plastics.

Table 1 shows the relationship between the thermal diffusivity of the fluids and the size of the semiconductor InP@ZnS nanoparticles. The results show that the thermal diffusivity of all the fluids containing semiconductor nanoparticles show lower values with respect to the base liquid toluene ( $\left.D=10.4 \mathrm{~cm}^{2} \cdot \mathrm{s}^{-1}[29]\right)$, so that the thermal diffusivity enhancement in semiconductor nanofluids is negative.

The minimum thermal diffusivity was obtained for the fluid containing semiconductor nanoparticles with lowest sizes. Although this interesting thermal behavior of nanofluids containing semiconductor nanoparticles has not been explored yet, below we propose a probable explanation to interpret such behavior. 


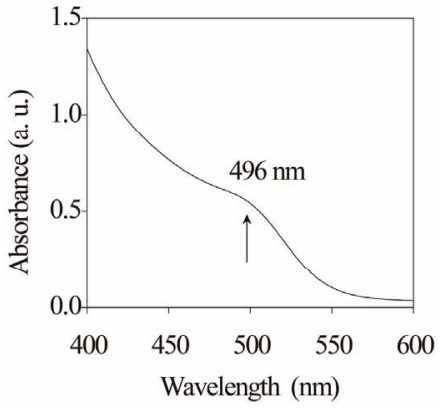

(a)

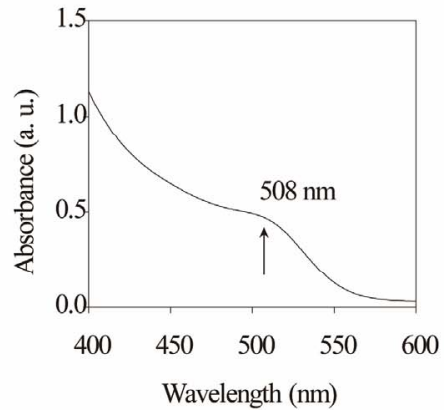

(b)

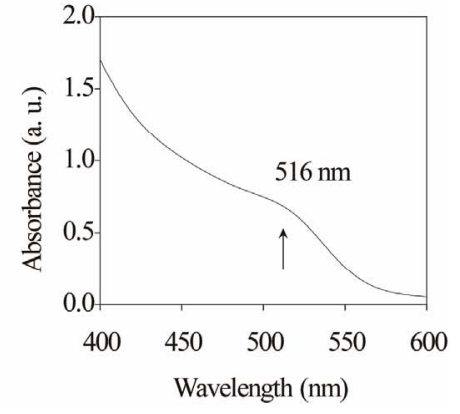

(c)

Figure 4. Optical absorption spectra of InP@ZnS semiconductor nanoparticles, (a) for S2 with absorption peak position of $496 \mathrm{~nm}$; (b) for S3 with absorption peak position of $508 \mathrm{~nm}$; and (c) for S4 with absorption peak position of $516 \mathrm{~nm}$.

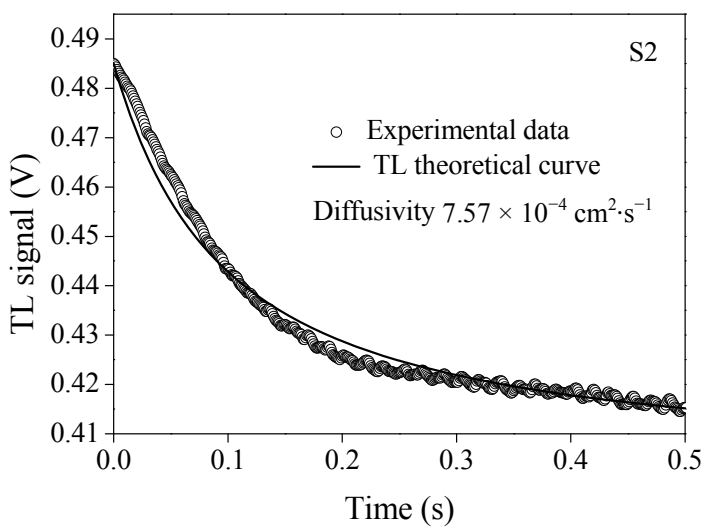

(a)

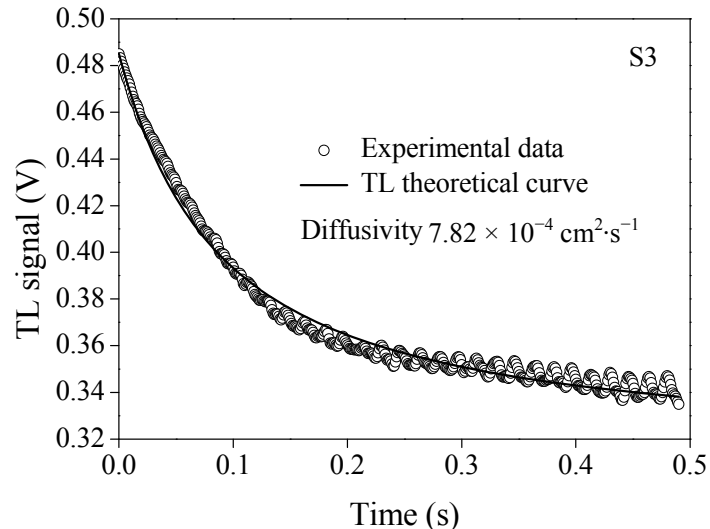

(b)

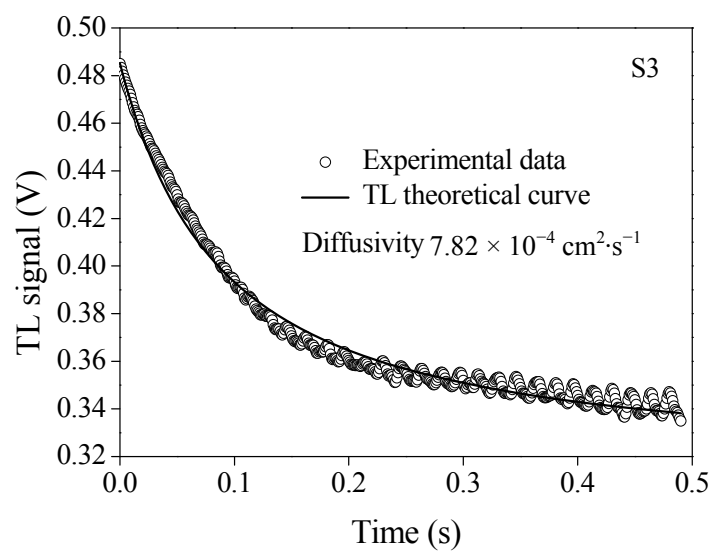

(c)

Figure 5. Time evolution of TL signal semiconductor InPn@ZnS nanoparticles with different size: (a) 3.1 nm; (b) $4.2 \mathrm{~nm}$; and (c) $4.6 \mathrm{~nm}$. Symbols (o) represent the experimental data and solid line, is the best fit to Eq.1.

Table 1. Thermal diffusivity of nanofluids for different sizes of the semiconductor InP@ZnS nanoparticles.

\begin{tabular}{ccc}
\hline InP@ZnS & Size $(\mathrm{nm})$ & Diffusivity $\left(10^{-4} \mathrm{~cm}^{2} \cdot \mathrm{s}^{-1}\right)$ \\
\hline S2 & 3.1 & 7.57 \\
S3 & 4.2 & 7.82 \\
S4 & 4.6 & 8.23 \\
\hline
\end{tabular}


Although this interesting thermal behavior of nanofluids containing semiconductor nanoparticles has not been explored yet, we propose a possible explanation to interpret such behavior.

There are recent studies about phonon transport in semiconductor nanostructures, more specifically analysis of semiconductor crystalline solids confined in the nanometer range in one, two or three dimensions. They report properties for heat transport of solid particles are of several orders of magnitude smaller than the corresponding bulk value $[21,22,30]$. For instance, for very thin silicon films and other nanostructures, the thermal conductivity models have shown that phonon confinement effects could affect thermal conduction by altering phonon spectra of the materials [31]. Theoretical studies [32] have suggested that in the extent $\mathrm{Si}$ nanowire diameter becomes smaller than $20 \mathrm{~nm}$, the phonon dispersion relation could be more easily modified thanks to phonon confinement. In this case, phonon group velocities would be significantly lower than bulk value. Experimental reports [33] have shown that thermal conductivity of silicon wires of nanometer diameter is two orders of magnitude smaller than the bulk value and has strong diameter dependence. This suggests that $\mathrm{ZnS}$ or InP semiconductor particles confined in nanometric scale would also show thermal conductivity (thermal diffusivity) that is significantly lower than that of corresponding bulk value. This is why we make an assumption that when semiconductor particles of InP, ZnS or with structure InP@ZnS confined at nanometer scale being dispersed in liquid bases such as toluene with higher thermal diffusivity, the effective thermal diffusivity of formed nanofluid can be significantly decreased. Experimental evidence of negative heat transport enhancement in nanofluids consisting of $2 \mathrm{~nm}$ titania semiconductor as nanoparticles dispersed in $50 \%(\mathrm{w} / \mathrm{w})$ water + ethylene glycol [34] support this hypothesis. Thus, while metallic and non-metallic nanoparticles play an important role in positive increment of thermal diffusivity for liquid bases, the InP, $\mathrm{ZnS}$ or InP@ZnS semiconductor nanoparticles confined at nanometric scale should contribute to decrease the thermal diffusivity. A possible mechanism of heat transport for such semiconductor nanofluids is described in Ref. [34]. This mechanism is related to the nature of heat transport in nanoparticles.

Besides nanoparticle type (metallic, non-metallic or semiconductor) and size, the structure type formed by InP@ZnS two different semiconductor nanostructures can play an important role on liquid's thermal properties. Though we could not define accurately why thermal diffusivity of semiconductor nanofluids has negative increment, the $D$ value of the nanofluids containing semiconductor nanoparticles InP@ZnS, certainly demonstrates the effect of the presence of semiconductor nanoparticles and the size on the reduction of $D$ in the semiconductor nanofluid.

\section{CONCLUSION}

InP@ZnS semiconductor nanoparticles of different sizes were obtained by using single-step procedure without precursor injection. Formation of nanocrystals with average 3.1, 4.2, and $4.6 \mathrm{~nm}$ in core-InP/shell- $\mathrm{ZnS}$ form was verified through TEM and HRTEM analysis. Optical absorption spectra along with EDS spectra revealed the formation and composition in the nanoparticles. The thermal diffusivity of toluene remarkably decreases due to the presence of InP@ZnS nanoparticles. Smaller thermal diffusivities were obtained for nanofluids containing smaller semiconducting nanoparticles. The decrease of thermal diffusivity of semiconducting nanofluids is probably consequence of negative increase of thermal diffusivity of semiconducting InP@ZnS type nanoparticles.

\section{ACKNOWLEDGEMENTS}

The authors are thankful to the Mexican Agencies, ICyTDF, CONACYT, COFAA-IPN, SIP-IPN for financial supports.

\section{REFERENCES}

[1] Choi, S.U.S. (1995) Developments and applications of non-Newtonian flows. American Society of Mechanical Engineers, New York, 99-105.

[2] Kakaç, S. and Pramuanjaroenkij, A. (2009) Review of convective heat transfer enhancement with nanofluids. International Journal of Heat and Mass Transfer, 52, 3187-3196.

[3] Wang, X.-Q. and Mujumdar, A.S. (2008) A Review on nanofluids, Part I: Theoretical and numerical investigations. Brazilian Journal of Chemical Engineering, 25, 613-630. doi:10.1590/S0104-66322008000400001

[4] Wang, X.-Q. and Mujumdar, A.S. (2008) A review on nanofluids, Part II: Experiments and applications. Brazilian Journal of Chemical Engineering, 25, 631-648. doi:10.1590/S0104-66322008000400002

[5] Wang, X.-Q. and Mujumdar, A.S. (2007) Heat transfer characteristics of nanofluids: A review. International Journal of Thermal Sciences, 46, 1-19. doi:10.1016/j.ijthermalsci.2006.06.010

[6] Kwek, D., Crivoi, A. and Duan, F. (2010) Effects of temperature and particle size on the thermal property measurements of $\mathrm{Al}_{2} \mathrm{O}_{3}$-water nanofluids. Journal of Chemical \& Engineering Data, 55, 5690-5695. doi:10.1021/je1006407

[7] Shima, P.D., Philip, J. and Raj, B. (2010) Synthesis of aqueous and nonaqueous iron oxide nanofluids and study of temperature dependence on thermal conductivity and viscosity. The Journal of Physical Chemistry C, 114, 18825-18833. doi:10.1021/jp107447q

[8] Xie, H. and Chen, L. (2011) Review on the preparation 
and thermal performances of carbon nanotube contained nanofluids. Journal of Chemical \& Engineering Data, 56, 1030-1041. doi:10.1021/je101026i

[9] Baby, T.T. and Sundara, R. (2011) Synthesis and transport properties of metal oxide docorated graphene dispersed nanofluids. The Journal of Physical Chemistry C, 115, 8527-8533. doi:10.1021/jp200273g

[10] Amiri, A., Shanbedi, M., Eshghi, H., Heris, S.Z. and Baniadam, M. (2012) Highly dispersed multiwalled carbon in water and experimental investigation of the thermophysical properties. The Journal of Physical Chemistry C, 116, 3369-3375. doi:10.1021/jp210484a

[11] Paul, G., Chopkar, M., Manna, I. and Das, P.K. (2010) Techniques for measuring the thermal conductivity of nanofluids: A review. Renewable \& Sustainable Energy Reviews, 14, 1913-1924. doi:10.1016/j.rser.2010.03.017

[12] Jiménez-Pérez, J.L., Sánchez-Ramírez, J.F., Cruz-Orea, A., Gutiérrez-Fuentes, R., Cornejo-Monroy, D. and LópezMuñoz, G.A. (2010) Heat transfer enhanced in water containing $\mathrm{TiO}_{2}$ nanospheres. Journal Nano Research, $\mathbf{9}$, 55-60.

[13] Jiménez-Pérez, J.L., Cruz-Orea, A., Sánchez-Ramírez, J.F., Sánchez-Sinencio, F, Martínez-Pérez, L. and López-Muñoz, G.A. (2009) Thermal characterization of nanofluids with different solvents. International Journal of Thermophys$i c s, 30,1227-1233$. doi:10.1007/s10765-009-0623-1

[14] Gutiérrez-Fuentes, R., Pescador-Rojas, J.A., Jiménez-Pérez, J.L., Sánchez-Ramírez, J.F., Cruz-Orea, A. and MendozaÁlvarez, J.G. (2008) Study of thermal diffusivity of nano fluids with bimetallic nanoparticles with $\mathrm{Au}$ (core)/Ag (shell) structure. Applied Surface Science, 255, 781-783. doi:10.1016/j.apsusc.2008.07.023

[15] Gutiérrez-Fuentes, R., Sánchez-Ramírez, J.F., JiménezPérez, J.L., Pescador-Rojas, J.A., Ramón-Gallegos, E. and Cruz-Orea. A. (2007) Thermal diffusivity determination of protoporphyrin IX solution mixed with gold metallic nanoparticles. International Journal of Thermophysics, 28, 1048-1055. doi:10.1007/s10765-007-0225-8

[16] Cahill, D.G., Ford, W.K., Goodson, K.E., Mahan, G.D., Maris, H.J., Merlin, R. and Phillpot, S.R. (2003) Nanoscale thermal transport. Journal Applied Physics, 93, 793.

[17] Andrey L.R. (2008) Semiconductor nanocrystal quantum dots. Synthesis, Assembly, Spectroscopy and Applications, Springer Wien, New York.

[18] El Sayed, M.A. (2004) Small is different: Shape-, size-, and composition-dependent properties of some colloidal semiconductor nanocrystals. Accounts of Chemical Research, 37, 326-333. doi:10.1021/ar020204f

[19] Walling, M.A., Novak, J.A. and Shepard, J.R.E. (2010) Quantum dots for live cell and in vivo imaging. International Journal of Molecular Sciences, 10, 441-491. doi:10.3390/ijms10020441

[20] Hussain, S., Won, N., Nam, J., Bang, J., Chung, H. and Kim, S. (2009) One-pot fabrication of high quality InP/ $\mathrm{ZnS}$ (core/shell) quantum dots and their applications to cellular imaging. ChemPhysChem, 10, 1466-1470.

[21] Liu, W. and Asheghi, M. (2004) Phonon-boundary scater- ring in ultrathin single-crystal silicon layers. Applied Physics Letters, 84, 3819-3821. doi:10.1063/1.1741039

[22] Li, D., Wu, Y., Kim, P., Shi, L., Yang, P. and Majumdar, A. (2003) Thermal conductivity of individual silicon nanowire. Applied Physics Letters, 83, 2934.

[23] Bindhu, C.V., Harilal, S.S., Nampoori, V.P.N. and Villabhan, C.P.G. (1998) Thermal diffusivity measurements in organic liquids using transient thermal lens calorimetry. Optical Engineering, 37, 2791-2794. doi: $10.1117 / 1.601825$

[24] Li, L. and Reiss, P. (2008) One-pot synthesis of highly luminescent $\mathrm{InP} / \mathrm{ZnS}$ nanocrystals without precursor injection. Journal of the American Chemical Society, 130, 11588-11589. doi:10.1021/ja803687e

[25] Sánchez-Ramírez, J.F., Jiménez-Pérez, J.L., Carbajal-Valdez, R., Cruz-Orea, A. and Gutiérrez-Fuentes, R. (2006) Thermal diffusivity measurements in fluids containing metallic nanoparticles using transient thermal lens. International Journal of Thermophysics, 27, 1181-1188. doi:10.1007/s10765-006-0084-8

[26] Shen, J., Lowe, R.D. and Snook, R. D. (1992) A model for $\mathrm{cw}$ laser induced mode-mismached dua-beam thermal lens. Chemical Physics, 165, 385-396. doi:10.1016/0301-0104(92)87053-C

[27] Ivanov, S.A., Piryatinski, A., Nanda, J., Tretiak, S., Zavadil, K.R., Wallace, W.O., Werder, D. and Klimov, V.I. (2007) Type-II core/shell CdS/ZnSe nanocrystals: Synthesis, electronic structures, and spectroscopic properties. Journal of the American Chemical Society, 129, 1170811719. doi: $10.1021 / \mathrm{ja} 068351 \mathrm{~m}$

[28] Burda, C., Chen, X., Narayanan, R. and El-Sayed, M. A. (2005) Chemistry and Properties of Nano crystals of Different Shapes. Chemical Review, 105, 1025-1102.

[29] Weast, R.C. (1987) CRC handbook of chemistry and physics. CRC Press, Boca Raton. doi:10.1021/cr030063a

[30] Ponomareva, I., Srivastava, D. and Menon, M. (2007) Thermal conductivity in thin silicon nanowires: Phonon confinement effect. Nano Letter, 7, 1155-1159. doi: $10.1021 / \mathrm{n} 1062823 \mathrm{~d}$

[31] Balandin, A. and Wang, K. (1998) Significant decrease of the lattice thermal conductivity due to phonon confinement in a free-standing semiconductor quantum well. Physical Review B, 58, 1544-1549. doi:10.1103/PhysRevB.58.1544

[32] Khitun, A., Balandin, A. and Wang, K.L. (1999) Modification of the lattice thermal conductivity in silicon quantum wires due to spatial confinement of acoustic phonons. Superlattice and Microstructures, 26, 181-193. doi:10.1006/spmi.1999.0772

[33] Li, D., Wu, Y.Y., Kim, P., Shi, L., Yang, P. and Majumdar, A. (2003) Thermal conductivity of individual silicon nanowires. Applied Physics Letters. 83, 2934-2936. doi:10.1063/1.1616981

[34] Teja, A.S., Beck, M.P., Yuan, Y. and Warrier, P. (2010) The limiting behavior of the thermal conductivity of nanoparticles and nanofluids. Journal of Applied Physics, 107, 114319. 